\title{
AN INTEGRATED ULTRASONIC SENSOR BASED SMART CANE FOR ASSISTING THE VISUALLY IMPAIRED
}

\author{
Rohan Mahajan \\ B.E. Department of Computer Engineering \\ Indira College of Engineering and Management \\ Pune, India
}

\author{
Amit Kumar \\ B.E. Department of Computer Engineering \\ Indira College of Engineering and Management \\ Pune, India
}

\author{
Dr. Poorna Shankar \\ Head of Department \\ Department of Computer Engineering \\ Indira College of Engineering and Management \\ Pune, India
}

\begin{abstract}
Smart cane is an innovative stick developed for visually impaired individuals for improved guidance in navigation. We are proposing a sophisticated blind stick that enables visually impaired individuals to navigate with ease by using advanced technology. This Blind Stick is combined with ultrasonic detector in conjunction with water sensing. This project primarily uses ultrasonic sensors to detect obstacles ahead using ultrasonic waves. On sensing obstacles, the sensor forwards the relevant data to the micro controller. The microcontroller processes the received data and calculates the distance of the obstacle from the stick. If the obstacle isn't that close the circuit does nothing. If the obstacle is close enough the micro controller sends a signal to put on a buzzer. As an Additional feature, it detects and alerts through a different buzzer when close to water and it allows the blind to detect if there is light or darkness in the room. The system provides a unique feature for helping the blind find their stick if they forget where they kept it. A wireless RF primarily based remote is employed for this purpose. On pressing the remote button, a buzzer sounds on the stick that helps the visually handicapped person to seek out their stick. Thus, this system eventually permits obstacle detection furthermore as finding the stick if misplaced by visually impaired individuals.
\end{abstract}

Keywords : Server, Monitoring, Services, Resources, Faults, Alerts.

\section{INTRODUCTION}

Despite all medical revolution happening in the last 20 years there are approximately 37 million visually impaired people present globally out of which 10 million people are in India. Considering their day to day issues and to improve their social life an innovative low cost walking aid called smart cane has been designed and using ultrasonic sensors, infrared sensors and microcontroller. The proposed device will help the blind people in identifying and alerting them in pits, water logs and obstacles. It has a GPS feature to send the SMS alert to the closed ones during the panic situation faced by the blind people. The RF sensors enabled in the device helps to raise alarms and locate the device when it is misplaced.

Ultra-Sound Sensors- "Ultrasonic sensors" are based on the measurement of the properties of acoustic waves with frequencies above the human audible range, often at roughly 40 $\mathrm{kHz}$. They generally operate by generating a high-frequency pulse of sound, then receiving and evaluating the properties of the echo pulse[1].

3 exclusive residences of the obtained echo pulse may be evaluated, for special sensing functions. they may be:

- Time of flight (for sensing distance)

- Doppler shift (for sensing velocity)

Amplitude attenuation (for sensing distance, directionality, or attenuation coefficient).

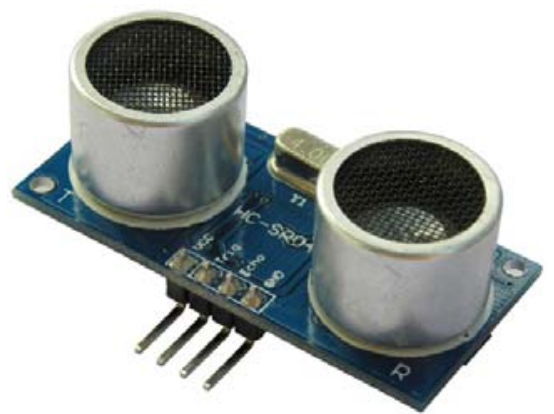

Fig. 1. Ultrasonic Sensor - HC-SR04

Modes Of Operation:

Time of Flight:

Reflection Mode -- In reflection mode (also referred to as "echo ranging"), an ultrasonic transmitter emits a brief burst of sound in a particular path. the pulse bounces off a goal and returns to the receiver after a time c program languageperiod $t$ [1]. The receiver records the length of this point interval, and calculates the space cosmopolitan $r$ based mostly all on the speed of sound c:. 


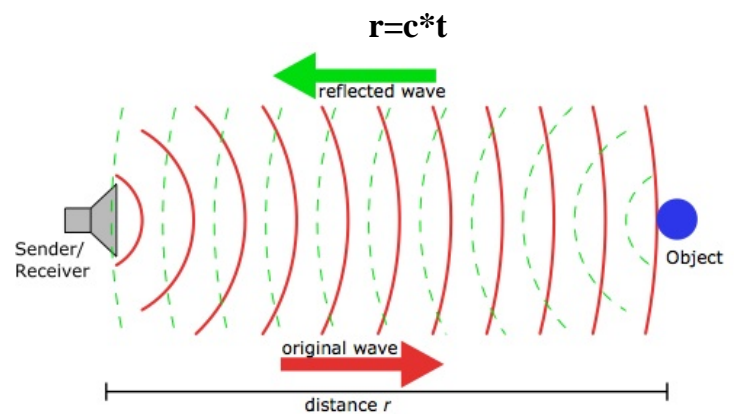

Fig. 2. Ultrasonic wave distance measure

Direct Measurement Mode:

In this mode of operation, the transmitter and receiver area unit 2 separate units that move relative to every alternative. for instance, the receiver may be fastened to a target that moves relative to a stationary transmitter, or vice-versa. Multiple transmitters may be accustomed increase the radial asymmetry of the transmitted pulse. placed multiple inaudible transmitters on a performing artist, whose signals were received by multiple receivers within the performance house, sanctioning a computer virus to triangulate the performer's position [1].

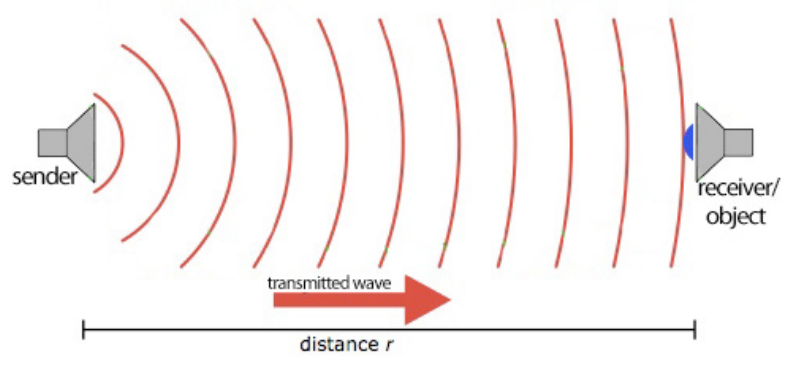

Fig. 3. Ultrasonic Wave

\section{Doppler Shift:}

When a wave reflects off of a moving object, its frequency is shifted by associate quantity proportional to the speed of the article. This reality is exploited in supersonic sensing by having the receiver live not the time of flight however the frequency of the returning echo pulse. Knowing iron and metallic element, the frequency of the emitted and received pulse, severally, the speed $\mathrm{v}$ of the target could also be calculated:

$$
f_{e}-f_{r}=2 f_{e}(v / c) \cos (A)
$$

where $\mathrm{A}$ is that the angle between the target's and also the pulse's lines of motion. [1]

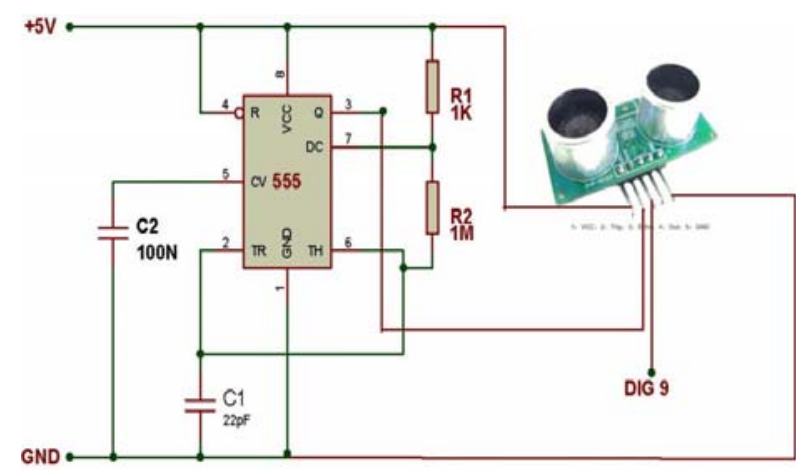

Fig. 4. Ultrasonic Sensor Circuit

\section{Advantages of Ultrasonic sensors:}

- the output price is linear with the space between the device and also the target
- sensor response is not smitten by the colors, transparency of objects, optical reflection properties,or by the surface texture of the issue

- these sensors square measure designed for contact-free detection

- sensors with digital (ON/OFF) outputs have wonderful repeat sensing accuracy

- $\quad$ accurate detection even of tiny objects

- ultrasonic sensors will add crucial conditions like dirt and dirt

- they are offered in cuboid or cylinder forms, that is healthier for a freedom style [2].

\section{Disadvantages of Ultrasonic sensors:}

- ultrasonic sensors should read a high-density surface permanently results. A soft surface like foam and artefact has density and absorb the sound waves emitted by the detector

- could have false responds for a few loud noises like air hoses

- $\quad$ the inaudible sensors have a latency with a fraction but alternative forms of sensors

- an inaudible detector incorporates a minimum sensing distance, that ought to be taken into thought after you opt for the detector

- $\quad$ some changes within the atmosphere will have an effect on the response of the detector (temperature, humidity, pressure, etc.) [3]

Infrared sensor: Associate in Nursing infrared detector measures the IR light-weight that's transmitted within the atmosphere to seek out objects by Associate in Nursing IR diode. this kind of detector is extremely standard in navigation for object turning away, distance measured or line following applications. This detector is extremely sensitive to IR lights and daylight, Associate in Nursingd this can be the most reason that an IR detector is employed with nice exactitude in lowlight[4].

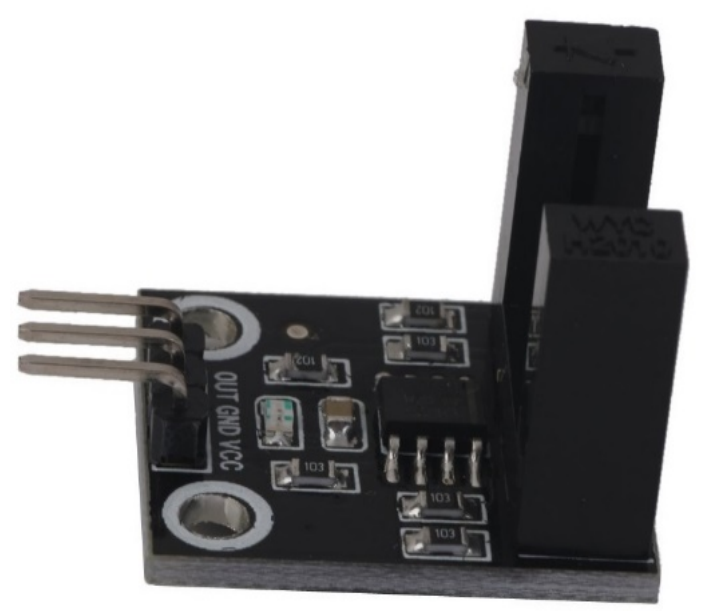

Fig. 5. Infrared Sensor 


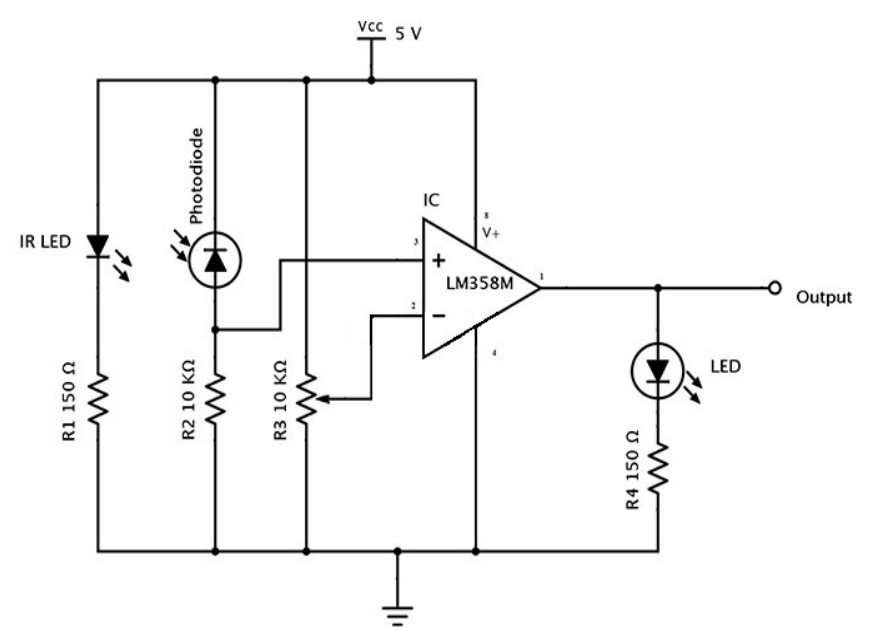

Fig. 6. IR Sensor Circuit

Advantages of Infrared sensors:

- $\quad$ can observe infrared radiation over an oversized space

- $\quad$ can operate in time period

- IR detector uses non-visible light-weight for detection are low cost sensors.[4]

\section{Disadvantages of Infrared sensors:}

- this detector is extremely sensitive to IR lights and daylight

- $\quad$ it has a weakness to darker colours like black [4].

\section{LITERATURE SURVEY}

Independence is the building methodology in achieving dreams, goals and objectives in life. Visually impaired persons find themselves challenging to go out independently. There are millions of visually impaired or blind people in this world who are always in need of helping hands. For many years the white cane became a widely known attribute to blind person's navigation and later efforts have been created to improve the cane by adding remote sensing element. Blind people have big problem when they walk on the street or stairs using white cane, but they have sharp haptic sensitivity. The electronic walking stick can facilitate the visually handicapped person by providing a lot of convenient means that of life. the most aim of this paper is to contribute our information and services to the folks of blind and disable society [5].

The paper presents a theoretical model and a system idea to offer a good electronic aid for blind individuals. The system is meant to offer the visually challenged a higher walking expertise. The design is incorporated with Ultrasonic sensors for Obstacle detection, supported with heat and water detection. Ultrasonic sensors are used to calculate distance of the obstacles around the blind person to guide the user towards the available path. This research work explains about the setup we used for the implementation, design details [6].

Blind people need some aid to feel safe while moving. Smart stick comes as a planned resolution to boost the quality of each blind and visually impaired folks. Stick resolution use totally different technologies like inaudible , infrared and optical maser however they still have drawbacks. In this paper we have a tendency to propose, light-weight weight, cheap, user friendly, quick response and low power consumption, sensible stick supported infrared technology. A pair of infrared sensors can detect stair-cases and other obstacles presence in the user path, within a range of two meters. The experimental results achieve good accuracy and the stick is able to detect all of obstacles [7].

\section{PROPOSED SYSTEM OUR PROPOSED SYSTEM HAS THE FOLLOWING FEATURES:}

\section{Obstacle Detection:}

The obstacle detection goal is accomplished by adding ultrasonic sensors at specific positions to the cane. After identifying the obstacles, the stick alerts the visually impaired people using vibration signals. Thus, we detect the objects in all directions and help blind people to be aware of their surronding.

\section{Pit Detection:}

The infrared sensor which is used to scan the ground around the blind person by emitting-reflecting waves.

\section{Buzzer Alert:}

A wireless RF based remote is used for this purpose. Pressing the remote button sounds a buzzer on the stick that helps the blind man to search out their stick.

\section{Panic Alert:}

The device is linked with a GPS to identify the location of the blind person. Moreover, for emergency a panic text is sent to the contacts with users location. Acknowledgment

\section{ARCHITECTURE}

Over sensing obstacles, the detector handovers this knowledge to the microcontrollers. The microcontroller processes this information and calculates if the obstacle is within proximity enough. If the obstacle isn't in enough proximity the circuit will do nothing. If the obstacle is in enough proximity the microcontroller sends an indication. A wireless RF primarily based remote is employed for this purpose [8]. Pressing the remote button sounds a buzzer on the stick which helps the blind person to find their stick Thus, this system allows for obstacle detection as well as finding stick if misplaced by visually disabled people can be accomplished using LOGS and various data mining algorithms [9]. The system includes active obstacle detection which will keep the user safe from colliding with them. The infrared sensors which is integrated on the lower end of the stick is provided with the intention to detect pits in proximity.

Advantages:

1. Aiding the visually challenged

2. Navigate through the streets.

3. Get obstacle proximity alert

4. Real Time Data Analysis

5. Easy to Use

6. Panic alert for emergencies.

7. Real time GPS navigation. 


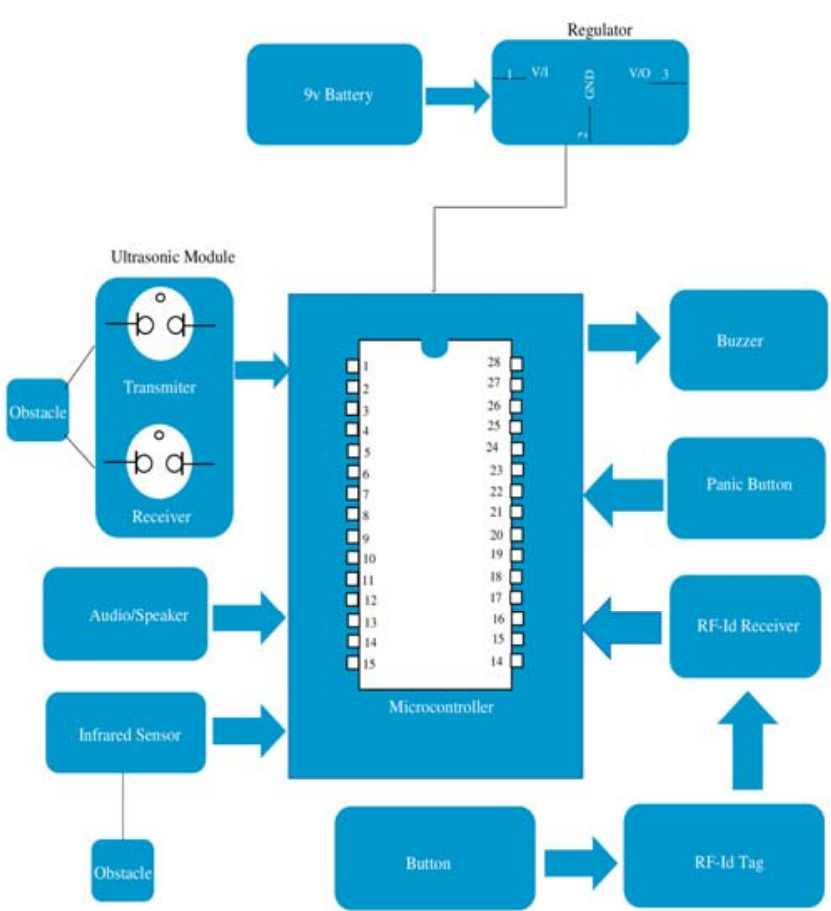

Fig 7. Block Diagram of System

Sate Diagram of Smart Cane:

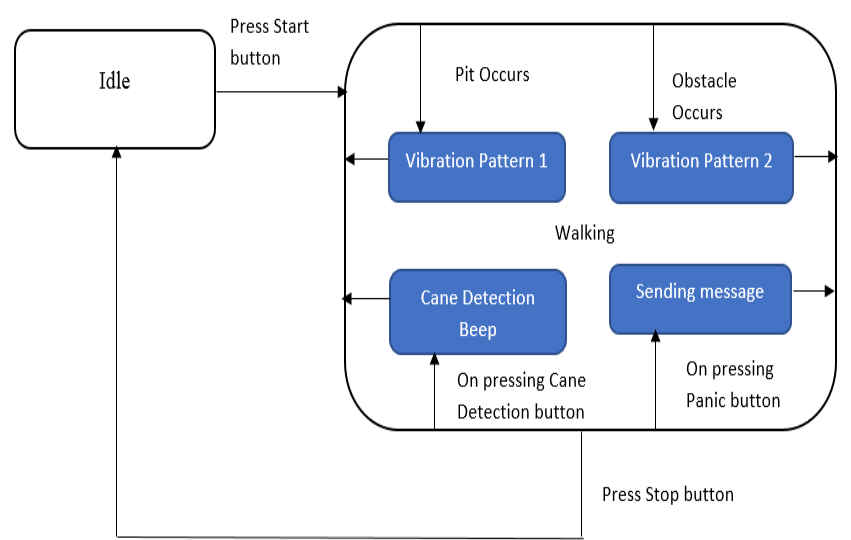

Fig 8. State Diagram of Smart Cane

\section{CONCLUSION}

All the studies which had been reviewed show that, there are a number of techniques for making an ultrasonic blind walking stick for blind people. The advantage of the system lies in the fact that it can prove to be a very low-cost solution to millions of blind persons worldwide. It has all the features to locate, identify and alert obstacles, pits, water logs and helps the blind during emergence situation. The smart white cane is a practically feasible product and convenient to carry around like any other walking sticks. This could also be considered a crude way of giving the blind a sense of vision.

\section{FUTURE SCOPE}

Future work includes installation of GPS system for navigation along with additional sensors like accelerometers, PIR motion detector and digital compass which tell the exact location of the user.

\section{REFERENCES}

[1] http://sensorwiki.org/doku.php/sensors/ultrasound

[2] http://nevonprojects.com/ultrasonic-blind-walking-stick-project/

[3] https://en.wikibooks.org/wiki/Robotics/Sensors/Ranging_Sensors

[4] https://www.intorobotics.com/types-sensors-target-detectiontracking/

[5] M.P. Menikdiwela, K.M.I.S. Dharmasena, A.M. Harsha S. Abeykoon, "Haptic based walking stick for visually impaired people”, Proc. Int. Conf. CCUBE, pp. 1-6, Dec. 2013.

[6] Ankit Agarwal, Deepak Kumar, Abhishek Bhardwaj, "Ultrasonic Stick for Blind”, IJCES, Volume 4 Issue 4, April 2015

[7] Shashank Chaurasia, K.V.N. Kavitha, “An Electronic Walking Stick For Blinds, International Conference on data Communication and Embedded Systems ICICES, pp. 1-5, 2014.

[8] Sudhanthiradevi.M, Suganya Devi.M,Roshini.R ,Sathya.T, “Arduino Based Walking Stick For Visually Impaired", IJARTET 2016

[9] Shubham Adhe, Sachin Kunthewad, Preetam Shinde,Mrs.V.S.Kulkarni "Ultrasonic Smart Stick for Visually Impaired People”, IOSR-JECE, NCIEST-2015 . 